\title{
Evaluación de la gestión de continuidad de los servicios: Universidad de Costa Rica, Sede de Occidente ${ }^{1}$
}

\author{
Evaluation of Service Continuity Management: \\ University of Costa Rica, Western Headquarters
}

Avaliação da gestão de continuidade dos serviços: a Universidade da Costa Rica, Sede Occidente

\section{Lisa Campos Pérez}

Universidad de Costa Rica. San Ramón, Costa Rica

lisa.campos@ucr.ac.cr

https://orcid.org/0000-0002-0191-7422

\section{Jorge Esquivel Arias}

Universidad de Costa Rica. San Ramón, Costa Rica jorgearturo.esquivel@ucr.ac.cr https://orcid.org/0000-0001-9169-0892

\section{Daniela Varela Rojas \\ Universidad de Costa Rica. San Ramón, Costa Rica daniela.varela@ucr.ac.cr \\ https://orcid.org/0000-0002-4549-1419}

DOI: https://doi.org/10.32719/25506641.2021.10.7

Recibido: 15 de octubre de 2020 - Revisado: 13 de noviembre de 2020

Aceptado: 9 de febrero de 2021 • Publicado: 1 de julio de 2021

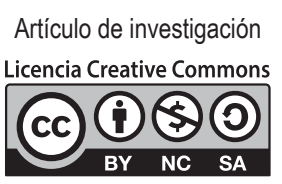

1. Artículo para publicación en el marco del Proyecto de Investigación de la Universidad de Costa Rica, VI 225-C0-206. 


\section{Resumen}

La Universidad de Costa Rica (UCR) es una de las cinco universidades públicas de la República de Costa Rica, la Sede de Occidente (UCR-SO) fue la primera sede regional de la UCR, fundada en 1968. En los últimos años se ha visto afectado el desarrollo de las actividades universitarias y el uso de sus instalaciones debido a fenómenos de diferente naturaleza que han afectado a la institución. El documento tiene como propósito evaluar el nivel de desarrollo de las estrategias de continuidad de la UCR-SO, mediante el análisis de los sistemas de gestión de riesgos y continuidad de los servicios esenciales de la institución, a través de la auditoría de cinco normas internacionales y factores internos, con lo cual se busca identificar oportunidades de mejora en relación con la continuidad institucional de la UCR-SO. Se obtuvo como resultado el poco desarrollo de los sistemas de gestión evaluados y la necesidad que presenta la institución de generar e integrar un modelo que asegure la prestación oportuna y de calidad de esos servicios, especialmente los catalogados como esenciales por su incidencia en el desarrollo educativo, social e investigativo; así como en el funcionamiento efectivo de la institución.

Palabras clave: servicios, gestión, riesgo, universidad pública, cultura organizacional.

JEL: I23 Centros de enseñanza superior y de investigación.

\section{Abstract}

The University of Costa Rica (UCR) is one of the five public universities of the Republic of Costa Rica, the Western Headquarters (UCR-SO) was the first regional headquarters of the UCR, founded in 1968. In recent years the development of university activities and the use of its facilities have suffered due to different phenomena that have affected the institution. The purpose of the document is to evaluate the development level of the continuity strategies of the UCR-SO, through the analysis of the risk management systems and the essential service continuity of the institution. Under the audit of five international standards and internal factors of the institution which seeks to identify improvement opportunities in relation to the Institutional Continuity of the UCR-SO. Obtaining as a result the little development of the evaluated management systems and the need that the institution features to generate and integrate a model ensuring the timely and quality provision of these services, especially in essential services due to their effect in educational, social and investigative development; as well as in the effective operation of the institution.

Keywords: services, management, risk, public university, organizational culture.

JEL: I23 Higher Education and research institutions.

\section{Resumo}

A Universidade da Costa Rica (UCR) é uma das cinco universidades públicas da República da Costa Rica, e a Sede Occidente (UCR-SO) foi a primeira sede regional da UCR, fundada em 1968. Nos últimos anos, fenômenos de diferentes naturezas que atingiram a instituição afetaram o desenvolvimento das atividades universitárias e o uso de suas 
instalações. O presente documento tem como propósito avaliar o nível de desenvolvimento das estratégias de continuidade da UCR-SO por meio da análise dos sistemas de gestão de riscos e continuidade dos serviços essenciais da instituição. Isso será realizado mediante uma auditoria interna de cinco normas internacionais e fatores internos da instituição, com o qual se pretende identificar oportunidades de melhoria em relação à Continuidade Institucional da UCR-SO. Como resultado, identificaram-se o pouco desenvolvimento dos sistemas de gestão avaliados e a necessidade por parte da instituição de gerar e integrar um modelo que assegure a prestação oportuna e de qualidade dos serviços, sobretudo serviços catalogados como essenciais por sua incidência sobre o desenvolvimento educativo, social e investigativo, assim como sobre o funcionamento efetivo da instituição.

Palavras chave: serviços, gestão, risco, universidade pública, cultura organizacional. JEL: I23 Ensino superior e centros de pesquisa.

\section{Introducción}

H

oy, más que nunca, ante la creciente activación de los fenómenos naturales, tecnológicos y sociales, se ha evidenciado la importancia de que las instituciones gestionen los riesgos que las puedan afectar. Producto de esto, las organizaciones se ven en la necesidad de establecer "una serie de medidas técnicas, organizativas y procedimentales que garanticen la continuidad de las actividades o procesos de negocio en caso de tener que afrontar una contingencia grave" (INTECO 2015, 7).

Esta gestión promueve la continuidad de los servicios mediante la reducción de los riesgos y la planificación de su recuperación, lo cual, en contextos de emergencia como el actual, constituye una herramienta que fomenta la creación de marcos institucionales de preparación, respuesta y recuperación ante situaciones imprevistas en procura de satisfacer las necesidades de los usuarios, mediante la definición de medidas que permitan continuar realizando las actividades estándar de la institución.

En Costa Rica, la Contraloría General de la República (CGR), para conocer e identificar el nivel de la gestión para la continuidad de los servicios públicos críticos ante la emergencia sanitaria, se encuentra ejecutando un seguimiento de la gestión de la continuidad de 21 servicios públicos críticos prestados por 90 instituciones públicas, marcando el camino para el aparato público. Ante esto, siendo la UCR una de las cinco universidades públi- 
cas del país, y por su reconocimiento en la región según la clasificación de universidades QS World University Ranking regional para América Latina (571-580 del ranking mundial) (2021), ubicándose en el puesto número veinte y cinco, tiene la responsabilidad de liderar y ser la institución de educación superior pionera a nivel nacional, en identificación de riesgos potenciales que puedan afectar la continuidad de los servicios y definición de medidas de respuesta que permitan reaccionar de manera eficiente y expedita ante un incidente, para garantizar el bienestar de sus usuarios.

La UCR-SO, ubicada en la Zona de Occidente de la Gran Área Metropolitana, corresponde a la primera sede regional de la UCR, fundada en 1968 con el recinto de San Ramón y, posteriormente, en 1977 se fundó el segundo recinto ubicado en Tacares de Grecia (Universidad de Costa Rica 2019).

La historia reciente deja ver una serie de incidentes que han afectado el desarrollo de las actividades universitarias y sus instalaciones. En la tabla 1 se presentan los datos correspondientes.

La UCR identifica, como uno de los factores que pueden afectar el desarrollo de las actividades sustantivas, a las deficiencias en la gestión de riesgos; por dicha razón, existe la necesidad de incluir programas que permitan identificar las vulnerabilidades de las actividades relacionadas a las funciones de la institución. Ante esto, la UCR-SO ha planteado el desarrollo de una serie de proyectos y actividades tales como: el diseño de un plan de operación anual y definición del presupuesto para la Comisión de Gestión de Riesgos y Atención de Emergencias (CGRAE), y el desarrollo del Plan de Emergencias UCR-SO 2018-2019.

A pesar de estos esfuerzos, la UCR-SO se ha visto afectada por la materialización de riesgos, afectando el desarrollo de las actividades propias del quehacer universitario y a los diferentes usuarios de la institución. Un ejemplo de esto es la afectación por la pandemia de la COVID-19, tal y como se muestra en la tabla 1. Dicho incidente impide la realización de actividades presenciales, obligando a la institución a la virtualización de sus servicios, para lo cual no se encontraba preparada, provocando pérdidas de matrícula y un cambio en el modelo de trabajo institucional. 
Tabla 1

Incidentes históricos UCR-SO

\begin{tabular}{|c|c|c|}
\hline Incidente & Período & Afectación \\
\hline Tornados y vientos & 9/10/2017-12/01/2018 & $\begin{array}{l}\text { - Daños estructurales. } \\
\text { - Cierre de la sección de Biología. } \\
\text { - Interrupción en el uso de los laboratorios. }\end{array}$ \\
\hline Huracán Otto & $\begin{array}{l}24 / 11 / 2016- \\
25 / 11 / 2016\end{array}$ & - Suspensión de actividades universitarias. \\
\hline \multirow{3}{*}{$\begin{array}{c}\text { Disturbios } \\
\text { o manifestaciones }\end{array}$} & $12 / 9 / 2017-13 / 9 / 2017$ & $\begin{array}{l}\text { - Cierre de la sede por manifestaciones. } \\
\text { - Suspensión de lecciones y actividades } \\
\text { universitarias. } \\
\text { - Atraso en labores administrativas. }\end{array}$ \\
\hline & 20/9/2017-28/9/2017 & $\begin{array}{l}\text { - Imposibilidad para utilizar el edificio } \\
\text { de Dirección de la sede. } \\
\text { - Interrupción de las labores administrativas. }\end{array}$ \\
\hline & 28/9/2019-22/10/2019 & $\begin{array}{l}\text { - Interrupción de las actividades } \\
\text { en los edificios de Ingeniería industrial } \\
\text { y conjunto de aulas } 200 \text {. } \\
\text { - Falta de espacios para el desarrollo } \\
\text { de las actividades académicas. } \\
\text { - Incumplimiento de contratos. }\end{array}$ \\
\hline Pandemia COVID-19 & 16/3/2020-actualidad & $\begin{array}{l}\text { - Imposibilidad de desarrollar actividades } \\
\text { de docencia, investigación y acción social } \\
\text { de manera presencial. } \\
\text { - Virtualización forzada. }\end{array}$ \\
\hline
\end{tabular}

Elaboración propia.

Lo anterior refleja la importancia de que las instituciones de educación superior propicien herramientas que permitan generar no solo una mejor respuesta institucional ante una emergencia o desastre, sino también garantizar la recuperación de las actividades en un período prudencialmente aceptable.

Con base en lo expuesto anteriormente, el objetivo del presente artículo es evaluar el estado actual de la UCR-SO, por medio del uso de herramientas para la identificación de oportunidades de mejora en materia de gestión de riesgos y continuidad de los servicios esenciales de la institución. 


\section{Revisión de la literatura}

\section{Normas de referencia}

Las normas son estándares que facilitan maneras de desarrollar una tarea de modo que satisfagan las necesidades de los usuarios. Estos estándares se establecen en torno a consensos de gran alcance y con la participación de comités técnicos cualificados.

En esta investigación se utiliza como herramienta una serie de normas, que sirven de referencia para el desarrollo de una solución que permita satisfacer las necesidades de la institución y se detallan a continuación:

- INTE/ISO 22301:2015. Seguridad en la sociedad. Sistemas de gestión de continuidad del negocio. Requisitos: esta norma especifica los requisitos para planificar, establecer, implementar, operar, dar seguimiento, revisar, mantener y mejorar continuamente un sistema de gestión documentado para protegerse, reducir la posibilidad de ocurrencia, prepararse, responder y recuperarse de los incidentes disruptivos cuando ocurran (INTECO 2015). Cabe resaltar que esta norma internacional es aplicable a cualquier tipo de organización.

- INTE/ISO TS 22317:2016. Seguridad de la sociedad. Sistema de gestión de continuidad del negocio. Directrices para el análisis de impacto del negocio (BIA): esta especificación técnica proporciona una guía detallada para el establecimiento, implementación y mantenimiento de un proceso de análisis de impacto al negocio (BIA), coherentes con los requisitos de la norma INTE/ISO 22301. Es aplicable a la realización de cualquier proceso del BIA, ya sea parte de un sistema de gestión de continuidad del negocio (SGCN) o el programa de continuidad del negocio (programa de $\mathrm{BC}$ ). En lo sucesivo, el programa de BC se entenderá ya sea como SGCN o programa BC (INTECO 2016).

- INTE/ISO 22320:2013. Seguridad de la sociedad. Gestión de emergencias. Requisitos para respuesta ante incidentes: esta norma internacional determina los requisitos mínimos para una respuesta efectiva ante incidentes y provee las bases para el mando y control, información operativa, coordinación y cooperación para una respuesta ante incidentes dentro de una organización. Esto incluye estructuras y procedimientos de mando y 
control en la organización, decisiones de apoyo, trazabilidad, gestión de la información e interoperabilidad (INTECO 2013).

- CNE-NA-INTE-DN-01:2014. Norma de planes de preparativos y respuesta ante emergencias para centros laborales o de ocupación pública. Requisitos: este reglamento establece los requisitos y contenidos del plan de preparativos y respuesta ante una emergencia, para que sean incorporados en los centros laborales y de ocupación pública como parte de su dinámica diaria. Su objetivo es contribuir a la reducción de la vulnerabilidad y la amenaza, así como el impacto negativo que tienen los desastres en los centros laborales y de ocupación pública (INTECO y CNE 2014).

- INTE/ISO 31000:2018. Gestión del riesgo. Directrices: esta norma recomienda que las organizaciones desarrollen, implementen y mejoren de manera continua un marco de referencia cuyo objetivo sea integrar el proceso de gestión del riesgo en los procesos de gobernanza, de estrategia y de planificación, de gestión, y de elaboración de informes, así como en las políticas, los valores y en la cultura de toda la organización (INTECO 2018).

\section{Estado de la cuestión}

En Costa Rica existen pocos esfuerzos en relación a la gestión de la continuidad de los servicios en las instituciones públicas, ya que según la CGR (2020) en el estudio "Seguimiento de la gestión para la continuidad de los servicios públicos críticos ante la emergencia sanitaria”, en el cual se analizaron 90 instituciones públicas que prestan 21 servicios públicos críticos, se obtuvo que solamente una de las entidades se encuentra en el nivel óptimo, siete en el nivel avanzado y 82 en básico e incipiente en la gestión de la continuidad institucional.

En el caso de las instituciones de educación superior a nivel internacional, se puede mencionar a la Universidad Autónoma de Baja California (UABC), con su Plan de Continuidad Académica, en el que se presenta un protocolo que permite migrar el desarrollo académico de la Universidad a una plataforma virtual, que entra en operación en caso de presentarse algún incidente que imposibilite el acceso a sus instalaciones, asegurando el acceso 
a las lecciones por parte de los estudiantes y evitando, de esta manera, los inconvenientes en la prestación del servicio (Espinosa Díaz et al. 2012).

Otro ejemplo de esfuerzos desarrollados en el ámbito de la respuesta a emergencias y la continuidad de las operaciones es el "Modelo de gestión integral de emergencias y plan de continuidad del negocio", desarrollado en la Universidad Nacional de Colombia, Sede Palmira, el cual busca generar un modelo de respuesta para situaciones de emergencia o desastre para la preservación de la integridad de los recursos tanto humanos, sociales, tecnológicos y materiales y minimizando pérdidas y/o daños a los bienes de la institución, así como lograr la recuperación y funcionamiento normales en el período más corto posible (Fontal Hernández 2014).

Por su parte, la UCR-SO cuenta con el Plan de Emergencias 2018-2019, elaborado en octubre de 2018 por el asesor y consultor senior de salud ocupacional y seguridad humana, Luis Cordero López. El Plan es uno de los esfuerzos desarrollados por la institución para plantear protocolos de respuesta a emergencias y gestión de riesgos. Además, este documento plasma la condición de exposición a eventos adversos y sus impactos en las instalaciones y espacio físico de la sede, define estrategias, actividades y responsabilidades que permiten intervenir en los factores que propician de una u otra manera el eventual daño, permitiendo a la administración activa de la organización tomar las decisiones más oportunas y eficientes ante estos riesgos en conformidad con la Ley 8488 y la norma CNE-NA-INTE-DN-01:2014 (UCR 2018).

Si bien esta investigación permite identificar e intervenir posibles focos de riesgo para la institución y definir estrategias para la disminución de la probabilidad de ocurrencia y su atención, no contempla las etapas posteriores al incidente y su recuperación, poniendo en incertidumbre la prestación de los servicios esenciales de la institución tras la ocurrencia de un incidente disruptivo. Esta situación tiene el potencial de afectar la reputación, la operación y las finanzas de la organización, es por esta razón que cada vez más las instituciones de educación superior son conscientes de la importancia de propiciar herramientas que permitan generar no solo una mejor respuesta institucional ante una emergencia o desastre, sino que permitan garantizar la recuperación de las actividades en un período prudencialmente aceptable. 
Los ejemplos de estas instituciones de educación superior marcan el camino que deben seguir otras instituciones, con la finalidad de proteger sus operaciones así como a la comunidad universitaria, de los riesgos asociados al desarrollo de sus actividades, mediante una estrategia que permita definir los mecanismos, procedimientos y estructura para minimizar los impactos de un posible suceso.

\section{Metodología}

La metodología permite identificar las actividades y herramientas clave para el éxito del desarrollo de la investigación, permitiendo enfocar todos los esfuerzos en la planeación y ejecución de las actividades.

El tipo de investigación corresponde a un análisis cuantitativo, con la cual se analiza el desarrollo de los modelos de gestión de la continuidad del servicio y gestión de riesgos y atención de incidentes en la UCR-SO. Para ello, se utilizó el diseño detallado en la tabla 2.

Tabla 2

\section{Metodología}

\begin{tabular}{|c|l|}
\hline Actividad & \multicolumn{1}{c|}{ Herramientas } \\
\hline $\begin{array}{c}\text { Determinación del estado actual } \\
\text { de la UCR-SO, en relación con la gestión } \\
\text { de riesgos y continuidad de los servicios }\end{array}$ & $\begin{array}{l}\text { 1. Auditoría de la norma INTE/ISO 22301:2015. } \\
\text { 2. Auditoría de la norma INTE/ISO TS } \\
\text { 22317:2016. }\end{array}$ \\
$\begin{array}{c}\text { 3. Auditoría de la norma INTE ISO 22320:2013. } \\
\text { 2014. }\end{array}$ \\
\hline $\begin{array}{c}\text { Evaluación de la cultura organizacional } \\
\text { de la UCR-SO en términos de gestión } \\
\text { de riesgos y atención de emergencias }\end{array}$ & $\begin{array}{l}\text { 1. Censo de clima organizacional. } \\
\text { 2. Grupo focal con los involucrados. } \\
\text { 3. Análisis FODA. }\end{array}$ \\
\hline $\begin{array}{l}\text { Identificación de servicios } \\
\text { esenciales de la institución }\end{array}$ & 1. Matriz multicriterio de clasificación de servicios. \\
\hline
\end{tabular}

Elaboración propia. 
La metodología se compone de dos actividades: la determinación de los niveles de desarrollo de los modelos de gestión de la institución en las cinco normas a evaluar, y la evaluación de los aspectos del clima organizacional que pueden afectar la efectividad de estrategias de continuidad de los servicios y atención y respuesta ante incidentes.

1. Para la determinación del estado actual de la UCR-SO, con relación a la gestión de riesgos y continuidad de los servicios, se utilizó como instrumento la auditoría, teniendo como indicador cuantitativo el nivel de cumplimiento de cada una de las normas a evaluar, tal como consta en la tabla 3 .

\section{Tabla 3}

\section{Indicadores}

\begin{tabular}{|c|c|c|}
\hline Norma & Justificación & Indicador \\
\hline $\begin{array}{c}\text { INTE/ISO } \\
22301: 2015\end{array}$ & $\begin{array}{l}\text { Permite medir el grado de establecimiento e imple- } \\
\text { mentación de un sistema de gestión documentado } \\
\text { para protegerse, reducir la posibilidad de ocurrencia, } \\
\text { prepararse, responder y recuperarse de los incidentes } \\
\text { disruptivos cuando ocurran. }\end{array}$ & $\%$ de cumplimiento \\
\hline $\begin{array}{c}\text { INTE/ISO TS } \\
22317: 2016\end{array}$ & $\begin{array}{l}\text { Especifica los requisitos mínimos para una respues- } \\
\text { ta efectiva ante incidentes y provee las bases para el } \\
\text { mando y control, información operativa, coordinación y } \\
\text { cooperación para una respuesta ante incidentes dentro } \\
\text { de una organización. }\end{array}$ & $\%$ de cumplimiento \\
\hline $\begin{array}{c}\text { INTE ISO } \\
22320: 2013\end{array}$ & $\begin{array}{l}\text { Permite cuantificar el establecimiento y la implemen- } \\
\text { tación del proceso de análisis de impacto en el nego- } \\
\text { cio (BIA) formal y documentado para la organización; } \\
\text { el cual es un requisito para la aplicación de la norma } \\
\text { INTE/ISO 22301:2015. }\end{array}$ & $\%$ de cumplimiento \\
\hline $\begin{array}{l}\text { CNE-NA-INTE- } \\
\text { DN-01: } 2014\end{array}$ & $\begin{array}{l}\text { Establece los requisitos y contenidos con los que debe } \\
\text { cumplir un plan de preparativos y respuesta ante emer- } \\
\text { gencias en centros laborales y de ocupación pública. }\end{array}$ & $\%$ de cumplimiento \\
\hline $\begin{array}{c}\text { INTE/ISO } \\
31000: 2018\end{array}$ & $\begin{array}{l}\text { Proporciona directrices para gestionar el riesgo al que } \\
\text { se enfrentan las organizaciones. La aplicación de estas } \\
\text { directrices pueden adaptarse a cualquier organización } \\
\text { y su contexto. }\end{array}$ & $\%$ de cumplimiento \\
\hline
\end{tabular}

Elaboración propia. 
Con el propósito de clasificar el nivel de desarrollo de las normas evaluadas, se utilizó la categorización desarrollada por la CGR (2020), dicha categorización sugiere que para un nivel óptimo los sistemas de gestión se deben encontrar de 90 a $100 \%$, para un nivel avanzado en 75 a $90 \%$, para el nivel intermedio se aplican entre el 50 a $75 \%$, para un nivel básico entre 25 a $50 \%$ y para el incipiente entre el 1 a un $25 \%$.

\section{Evaluación de la cultura organizacional de la UCR-SO en términos de gestión de riesgos y atención de emergencias}

La evaluación de la cultura organizacional de la UCR-SO, en términos de gestión de riesgos y atención de emergencias, se aplicó a la totalidad de los funcionaros de las diferentes áreas y departamentos. Se desarrolló con el propósito de conocer la opinión y percepción de los colaboradores en relación con la gestión de riesgos y manejo de emergencias, e identificar oportunidades de mejora en relación a este aspecto; evalúa cinco aspectos que son de vital importancia en la gestión de la continuidad institucional y la respuesta y atención de emergencias. Las dimensiones se encuentran detalladas en la tabla 4.

Tabla 4

Aspectos a medir en el censo de clima organizacional

\begin{tabular}{|c|l|}
\hline Dimensión & \multicolumn{1}{c|}{ Definición } \\
\hline $\begin{array}{c}\text { Resistencia } \\
\text { al cambio }\end{array}$ & $\begin{array}{l}\text { Cambio entendido como a "cualquier pensamiento o acción dirigida contra un } \\
\text { cambio" (Harrinton, Conner y Horney, citado en Chacón 2017, 12). }\end{array}$ \\
\hline Liderazgo & $\begin{array}{l}\text { Conjunto de habilidades que permiten influir en un grupo y dirigirlo hacia el } \\
\text { logro de un objetivo o un conjunto de metas (Robbins y Judge 2013). }\end{array}$ \\
\hline $\begin{array}{c}\text { Fuerzas } \\
\text { motivacionales y } \\
\text { sentido } \\
\text { de pertenencia }\end{array}$ & $\begin{array}{l}\text { Se define la motivación como los procesos que inciden en la intensidad, di- } \\
\text { rección y persistencia del esfuerzo que realiza una persona para alcanzar un } \\
\text { objetivo (Robbins y Judge 2013). Por otra parte, el sentido de pertenencia } \\
\text { se refiere a un sentimiento de arraigo e identificación de un individuo con un } \\
\text { grupo o un ambiente determinado, su existencia genera en la persona un com- } \\
\text { promiso con la construcción de significados que a la larga formarán parte de la } \\
\text { memoria personal y del grupo al que se quiere pertenecer (Brea 2014). }\end{array}$ \\
\hline
\end{tabular}




\begin{tabular}{|c|l|}
\hline Dimensión & \multicolumn{1}{c|}{ Definición } \\
\hline Comunicación & $\begin{array}{l}\text { Es un instrumento sumamente útil para la aproximación entre personas, para } \\
\text { la resolución de problemas, para recibir información del medio, para reducir la } \\
\text { incertidumbre y, en definitiva, para el intercambio entre individuos y el medio } \\
\text { (Solano s. f.). }\end{array}$ \\
\hline $\begin{array}{c}\text { Condiciones físicas } \\
\text { de trabajo }\end{array}$ & $\begin{array}{l}\text { Es el conjunto de variables que definen la realización de una tarea en un en- } \\
\text { torno determinando la salud del trabajador en función de tres variables: fisica, } \\
\text { psicológica y social. Condiciones físicas: termorregulación, iluminación, espa- } \\
\text { cio y ruido (Unión General de Trabajadores 2020). }\end{array}$ \\
\hline
\end{tabular}

Fuente: Harrinton, Conner y Horney, citado en Chacón (2017, 12); Robbins y Judge (2013); Brea (2014); Solano (s. f.); Unión General de Trabajadores (2020).

A partir de las dimensiones expuestas en la tabla 4, se desarrollaron tres ítems para medirlas, con el propósito de que los participantes del censo califiquen el enunciado basado en la siguiente escala Likert:

Tabla 5

\section{Escala de Likert}

\begin{tabular}{|c|c|c|c|c|}
\hline \multicolumn{5}{|c|}{ Valoración } \\
\hline $\mathbf{1}$ & $\mathbf{2}$ & $\mathbf{3}$ & $\mathbf{4}$ & $\mathbf{5}$ \\
\hline $\begin{array}{c}\text { Totalmente } \\
\text { en desacuerdo }\end{array}$ & En desacuerdo & Neutral & De acuerdo & $\begin{array}{r}\text { Totalmente } \\
\text { de acuerdo }\end{array}$ \\
\hline
\end{tabular}

Elaboración propia.

Para efectos del análisis de las respuestas en cada variable, se definió en conjunto con la Comisión de Gestión de Riesgos y Atención de Emergencias de la UCR-SO establecer una meta de aceptación del $80 \%$ en cada una de las dimensiones a evaluar. Por el contrario, valores menores a dicho valor son considerados como oportunidades de mejora para el clima organizacional de la institución. 


\section{Identificación de servicios esenciales de la institución}

Con relación a la identificación de servicios esenciales de la institución, se evaluó el nivel de impacto de la interrupción de los servicios dentro de la institución. Es importante contemplar el contexto y la naturaleza de la institución, por lo que, con el propósito de tener una mayor claridad de los impactos que pueda tener una interrupción en el quehacer universitario, se realizó un análisis de los factores que componen un servicio esencial para la UCR-SO. A continuación, en la tabla 6 se presenta un diagrama de los factores y criterios de esencialidad definidos.

Tabla 6

Factores y criterios de esencialidad

\begin{tabular}{|c|l|c|}
\hline Variable & \multicolumn{1}{|c|}{ Definición } & \multicolumn{1}{c|}{ Fuente de información } \\
\hline Misionalidad & $\begin{array}{l}\text { Medida en que la no prestación de un ser- } \\
\text { vicio afecta la consecución de los objetivos } \\
\text { misionales de la institución. }\end{array}$ & $\begin{array}{l}\text { Calificación de 1-5 por parte de los } \\
\text { dueños del servicio mediante un } \\
\text { formulario de registro de servicios. }\end{array}$ \\
\hline Operatividad & $\begin{array}{l}\text { Mide características de cada uno de los ser- } \\
\text { vicios considerando aspectos tales como la } \\
\text { interdependencia, demanda, la percepción } \\
\text { del usuario (calidad) y la adaptabilidad (resi- } \\
\text { liencia) de los diferentes servicios que brin- } \\
\text { da la institución. }\end{array}$ & $\begin{array}{l}\text { Calificación de 1-5 por parte de los } \\
\text { dueños del servicio mediante un } \\
\text { formulario de registro de servicios. }\end{array}$ \\
\hline Presupuesto & $\begin{array}{l}\text { Mide la proporción del presupuesto que uti- } \\
\text { liza cada una de las áreas funcionales de la } \\
\text { institución, con el propósito de cuantificar la } \\
\text { cantidad de recursos que se destinan a cada } \\
\text { uno de los sectores de la institución y a los } \\
\text { servicios que ofrecen. }\end{array}$ & $\begin{array}{l}\text { Presupuesto de la UCR-SO 2020 } \\
\text { publicado por la Oficina de Planifi- } \\
\text { cación Universitaria. }\end{array}$ \\
\hline
\end{tabular}

Elaboración propia.

Para definir la esencialidad de los servicios identificados, se utilizó una matriz multicriterio con el propósito de ponderar el peso de cada uno de los criterios definidos anteriormente. Para esto, se utilizó una escala del uno al cinco para dar una calificación en los criterios de misionalidad, operatividad 
y presupuesto en cada uno de los servicios que ofrece la UCR-SO. La ponderación de los criterios de esencialidad utilizada en la matriz multicriterio se encuentra detallada en la figura 1.

Figura 1

Ponderación de los criterios de esencialidad

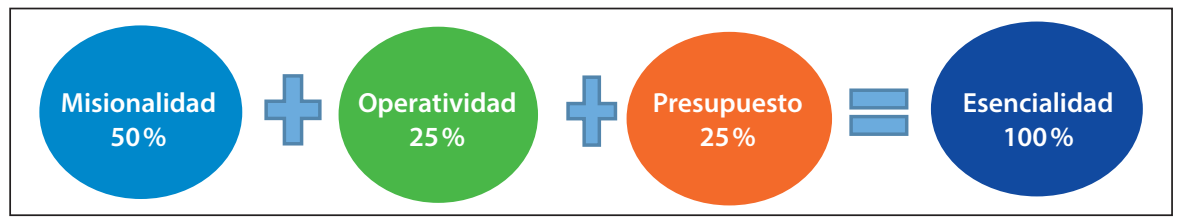

Elaboración propia.

\section{Resultados}

\section{Auditoría de normas}

En esta sección, se presentan los resultados de la evaluación realizada a la UCR-SO, en relación con la gestión de riesgos, atención de emergencias y continuidad de los servicios en la institución.

Figura 2

Resultados de evaluación a la norma INTE/ISO 22301:2015

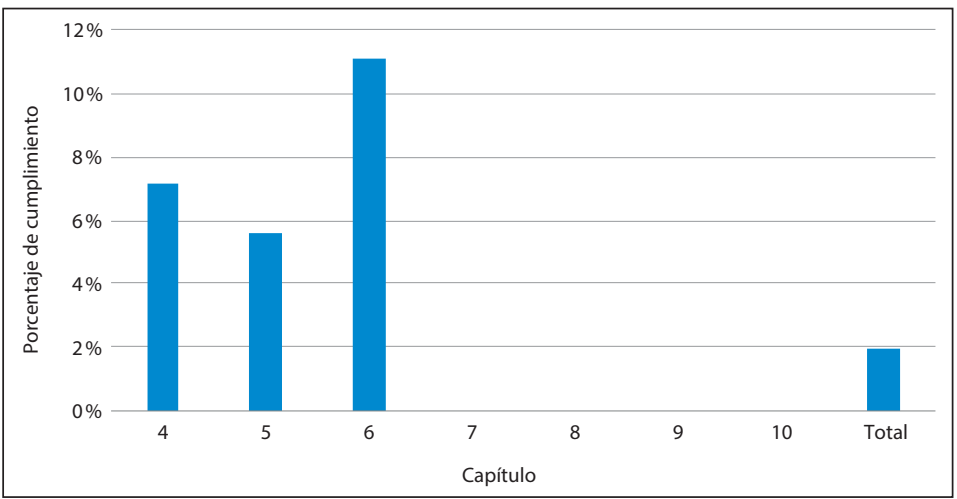

Elaboración propia. 
Con los resultados obtenidos, con relación a la norma INTE/ISO 223 01:2015 se logró identificar que la UCR-SO, sobre la continuidad de los servicios, se encuentra en un nivel de desarrollo incipiente, ya que solo cuenta con un porcentaje total de cumplimiento del $2 \%$. Esto se debe a que no cuenta con un sistema de gestión de continuidad de los servicios como tal; sin embargo, existen pequeños avances en conocimiento del contexto, liderazgo, planificación y soporte, gracias al trabajo de la CGRAE de la institución.

Figura 3

Resultados de evaluación norma INTE/ISO TS 22317:2016

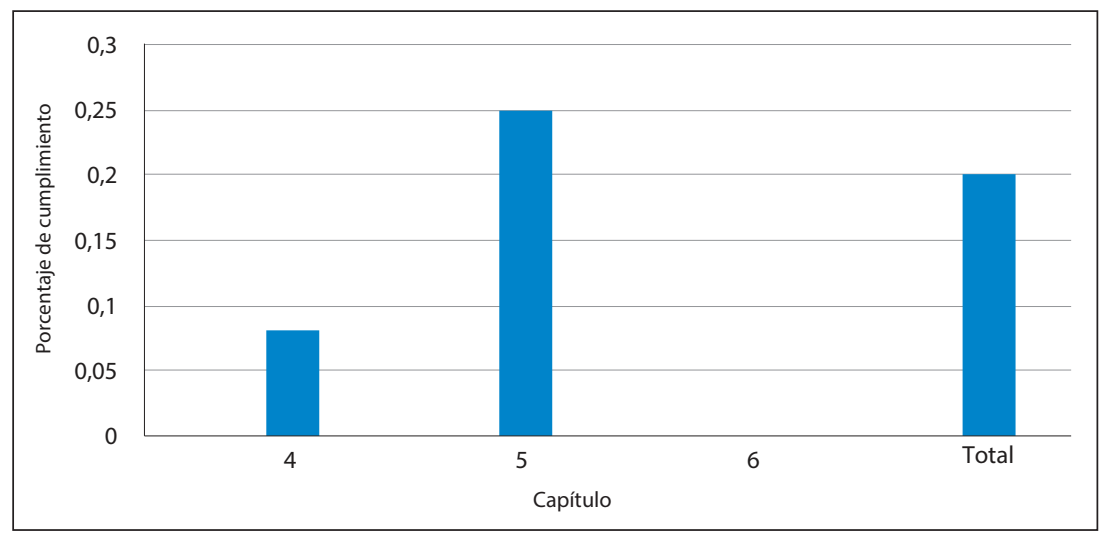

Elaboración propia.

Los resultados de la auditoría a la norma INTE/ISO TS 22317:2016 revelan un $20 \%$ de cumplimiento total, lo cual coloca a la institución en un nivel incipiente de desarrollo, esto se debe a que aún no se ha realizado un análisis de impacto sobre los servicios que ofrece la institución; sin embargo, existen algunos avances gracias a los esfuerzos que realiza la CGRAE de la institución y a diferentes insumos encontrados en documentación relacionada. 
Figura 4

Resultados de la evaluación a la norma INTE/ISO 22320:2013

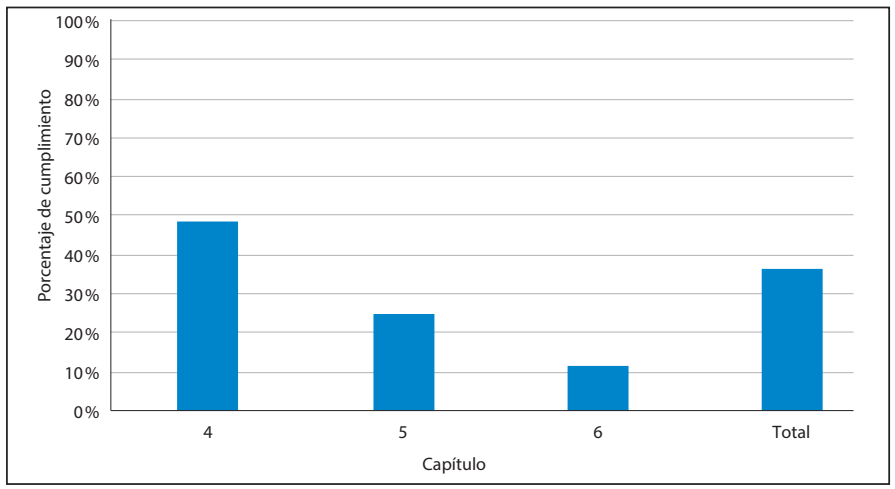

Elaboración propia.

Respecto a la respuesta ante incidentes, los resultados de la evaluación a la norma INTE/ISO 22320:2013, la UCR-SO se encuentra en un nivel básico de desarrollo, ya que la auditoría realizada muestra un $36 \%$ de cumplimiento total. La institución ha realizado grandes esfuerzos en el tema de gestión de emergencias; sin embargo, es necesario contar con un sistema estandarizado y más robusto, que permita dar una respuesta eficiente ante la presencia de un incidente disruptivo.

Figura 5

\section{Resultados de evaluación a la norma CNE-NA-INTE-DN-01:2014}

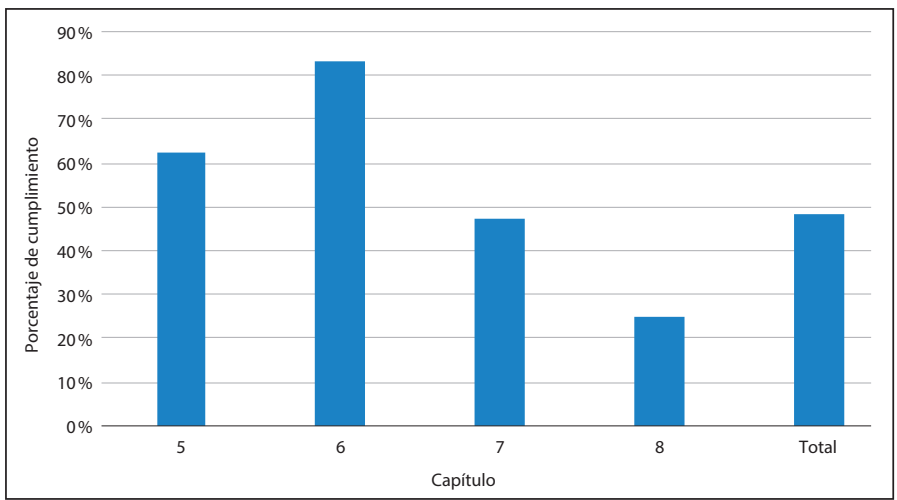

Elaboración propia. 
Con relación a los planes de preparativos y respuesta ante emergencias, según la evaluación a la norma CNE-NA-INTE-DN-01:2014, la UCR-SO se encuentra en un nivel básico de desarrollo con un $48 \%$ de cumplimiento total en la auditoría realizada. La institución cuenta con un plan de emergencias y diferentes esfuerzos por parte de la CGRAE; sin embargo, algunos de los insumos evaluados se encuentran incompletos y les falta un mayor grado de robustez para responder de manera adecuada ante una situación de emergencia.

Figura 6

\section{Resultados de evaluación a la norma INTE/ISO 31000:2018}

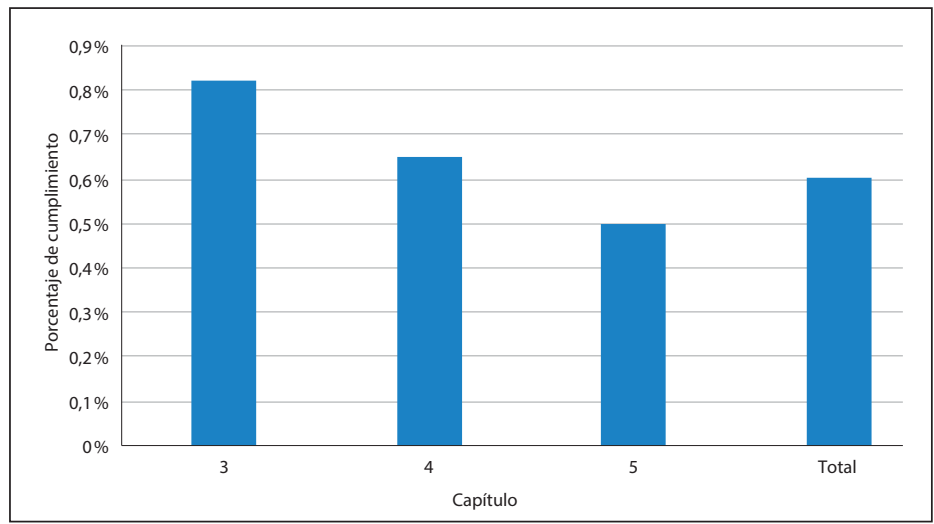

Elaboración propia.

Finalmente, con relación a la gestión de riesgos y evaluación a la norma INTE/ISO 31000:2018, la UCR-SO obtiene un 60\% de cumplimiento total, colocándola en un nivel intermedio de desarrollo. Dentro de los hallazgos más significativos, se encuentra el compromiso de la organización por incluir los principios de gestión de riesgos en las actividades de la organización; sin embargo, hay deficiencias en la operatividad, ya que los procesos no se encuentran adecuadamente definidos, lo cual dificulta la aplicación sistemática de políticas, procedimientos y prácticas a las actividades operativas de la gestión de riesgos. 


\section{Censo clima organizacional}

A continuación, se presentan los datos extraídos del análisis de clima organizacional para la UCR-SO en relación con la gestión de riesgos y el manejo de emergencias:

Figura 7

\section{Resultados del censo del clima organizacional}

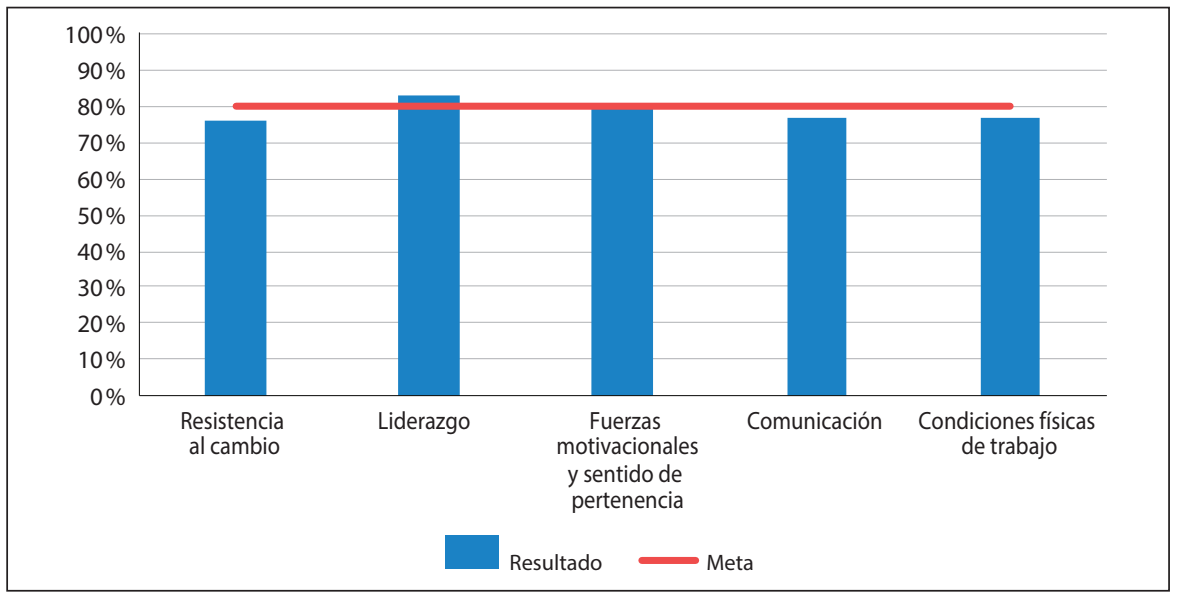

Elaboración propia.

La figura 7 resume los resultados del censo realizado a los encargados de cada coordinación o departamento. Como se puede observar, la variable de liderazgo muestra una mayor aceptación en las preguntas planteadas con un $83 \%$, seguida de la variable de fuerzas motivacionales y sentido de pertinencia que alcanza un $80 \%$. Ambas se consideran aceptables según el límite planteado en la metodología.

Por otra parte, las variables de comunicación y condiciones físicas de trabajo presentan un $77 \%$ de aceptación y resistencia al cambio un $76 \%$. Dichas variables son las más representativas respecto a oportunidades para la cultura organizacional y en específico para el desarrollo de programas de continuidad de los servicios, atención y respuesta ante incidentes. 


\section{Análisis FODA}

Como parte de la evaluación de la cultura organizacional de la UCR-SO, respecto a la gestión de riesgos y atención de emergencias, se realiza un análisis FODA que contempla los resultados del censo realizado e involucra a diferentes colaboradores y a los miembros de la CGRAE de la institución.

La matriz FODA se presenta a continuación:

\section{Tabla 7}

\section{Resultados análisis FODA}

\begin{tabular}{|c|c|}
\hline Fortalezas & Debilidades \\
\hline $\begin{array}{l}\text { - Liderazgo asertivo. } \\
\text { - Sentido de identidad y pertenencia a la institución. } \\
\text { - Interés y coordinación de los encargados de la CGRAE. } \\
\text { - Disposición de planes y documentos operativos con res- } \\
\text { - Vecto a gestión de riesgos y atención de emergencias. } \\
\text { - Delegación de funciones a nivel operativo y buena res- } \\
\text { puesta por parte de las brigadas de atención a emergen- } \\
\text { cias. }\end{array}$ & $\begin{array}{l}\text { - Falta de dirección. } \\
\text { - Canales de comunicación defi- } \\
\text { cientes. } \\
\text { - Cargas y políticas de trabajo } \\
\text { institucionales que provocan } \\
\text { poco involucramiento. } \\
\text { - Fuerte resistencia al cambio. } \\
\text { - Falta de conciencia colectiva. }\end{array}$ \\
\hline Oportunidades & Amenazas \\
\hline $\begin{array}{l}\text { - Implementación de programas de teletrabajo pertinente y } \\
\text { que se ajuste a las especificaciones docentes y administra- } \\
\text { tivas. } \\
\text { - Diseño, evaluación e implementación de un plan para ga- } \\
\text { rantizar la continuidad de los servicios. } \\
\text { - Creación de alianzas con instituciones públicas y privadas } \\
\text { para capacitaciones en temas de continuidad de los servi- } \\
\text { cios. }\end{array}$ & $\begin{array}{l}\text { - Opinión pública sin información } \\
\text { oportuna y veraz, puede lesio- } \\
\text { nar la imagen institucional. } \\
\text { - Recortes presupuestarios. } \\
\text { - Decisiones e intereses políti- } \\
\text { cos, que pueden afectar la di- } \\
\text { námica institucional. }\end{array}$ \\
\hline
\end{tabular}

Elaboración propia.

Para analizar los resultados que se recopilaron en el censo del clima organizacional se realizó un grupo focal, el cual consistió en reunir a los miembros de la comisión de gestión de riesgos y atención a emergencias de la UCR-SO, quienes tienen la experiencia necesaria para identificar las 
oportunidades, fortalezas, debilidades y amenazas en materia de gestión de riesgos y atención de emergencias; en total se consolidaron 16 hallazgos.

\section{Identificación de los servicios esenciales}

Para la clasificación de los servicios se utilizó la metodología $\mathrm{ABC},{ }^{2}$ para la cual se definió que los servicios que obtuvieran una calificación total igual o mayor al $80 \%$ de la calificación máxima (4), se clasifican como A; los servicios que lograran una calificación entre el $80 \%$ (4) y el 20\% (2) de la calificación máxima, se definen como categoría $\mathrm{B}$ y los servicios cuya calificación total sea menor al 20\% (2) de la calificación máxima, tienen categoría C. A partir de esto, se realizó la matriz multicriterio para los 94 servicios identificados, obteniendo como resultado 25 servicios tipo A, 67 tipo B y 2 tipo C.

Figura 8

\section{Clasificación de tipo de servicios por coordinación}

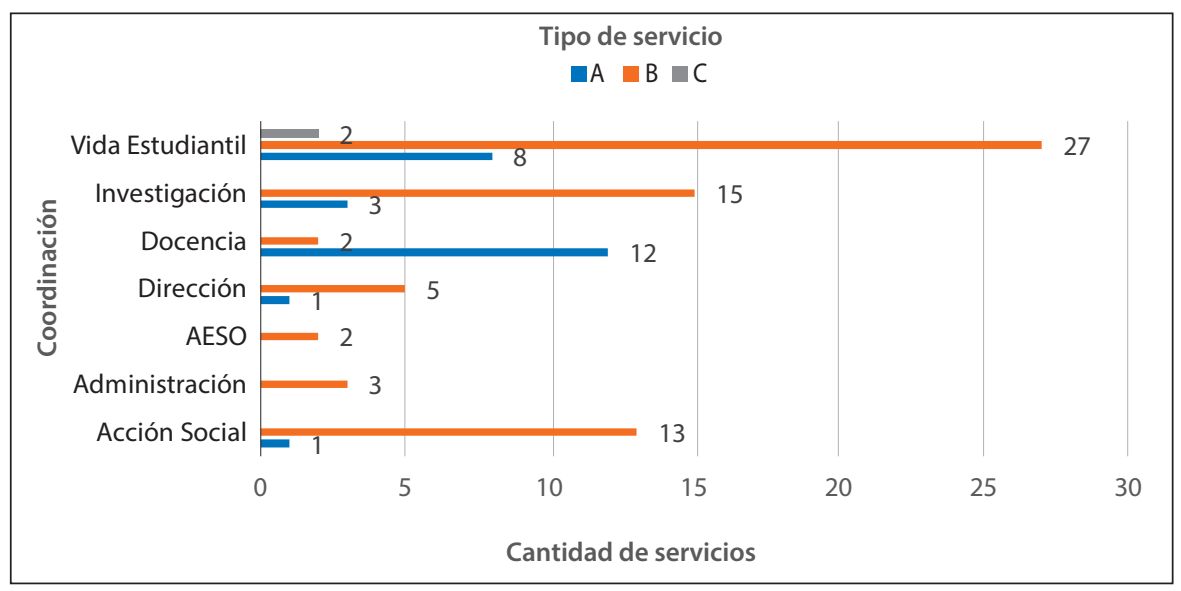

Elaboración propia.

2. Metodología de segmentación de acuerdo con criterios preestablecidos (indicadores de importancia); en este caso se utilizó el Principio de Pareto, también conocido como la Regla del $80 / 20$, asumiendo que los servicios esenciales (A) deben tener una calificación mayor al $80 \%$ de la valoración máxima, los servicios con valoraciones menores se categorizan como $\mathrm{B}$ y $\mathrm{C}$. 
En la figura 8 se puede observar que los servicios A se encentran distribuidos en las coordinaciones de docencia, vida estudiantil, investigación, dirección y acción social. Por su parte, los 67 servicios con clasificación B están distribuidos en todas las coordinaciones bajo estudio y la coordinación de vida estudiantil es la única que tiene servicios con clasificación C.

Según lo anterior, los 25 servicios tipo A son los esenciales de la UCR$\mathrm{SO}$, es decir, son absolutamente necesarios para mantener la actividad universitaria, esto con base en el impacto que tiene su no prestación sobre el cumplimiento de la misión de la organización, la operatividad de la universidad y el presupuesto destinado a cada uno de ellos. Por lo tanto, se deben enfocar los esfuerzos para protegerlos mediante un plan de continuidad, de manera que se garantice su recuperación a un nivel aceptable, presentando la menor afectación posible sobre la comunidad universitaria en caso de presentarse un incidente disruptivo.

\section{Conclusiones}

Tras el desarrollo de las auditorías de las normas, se logró identificar que la UCR-SO carece de sistemas de gestión que le permita manejar las políticas y los procedimientos de la organización con relación a manejo de riesgos, atención de emergencias y continuidad de los servicios de manera eficaz. Por lo tanto, se evidencia la necesidad de que la organización mejore su estructura con referencia a estos aspectos, de manera que se pueda minimizar la afectación de un incidente disruptivo sobre la continuidad de los servicios de la organización y los componentes de la comunidad universitaria.

Aunado a lo anterior, existe la posibilidad de que la UCR-SO sea una institución de educación superior pionera en la aplicación de estrategias de continuidad de los servicios, alineando sus esfuerzos con las regulaciones por parte de la CGR mediante el proyecto de "seguimiento de la gestión para la continuidad de los servicios públicos críticos ante la emergencia sanitaria", como un esfuerzo para asegurar la continuidad de los servicios públicos. El involucramiento de la UCR-SO en la creación de estrategias de continuidad tiene efectos directos sobre la calidad del servicio, logrando de esta manera 
generar la confianza y respaldo de la comunidad universitaria y la población en general.

La identificación de los servicios esenciales de la UCR-SO permite identificar los puntos clave en los cuales la organización debe generar estrategias de continuidad de manera que su afectación sea la mínima durante un evento disruptivo, protegiendo el acceso de la población a los servicios y los intereses de la institución mediante un plan de continuidad institucional.

Finalmente, con base en los hallazgos encontrados, se logra identificar la necesidad de contar con sistemas de continuidad estandarizados que sean sostenibles a lo largo del tiempo. Además, el involucramiento y la resiliencia de la comunidad universitaria son factores indispensables para el éxito del proyecto.

\section{Glosario}

Servicio: "Todas aquellas actividades identificables, intangibles, que son el objeto principal de una operación que se concibe intangibles, que son el objeto principal de una operación que se concibe para proporcionar la satisfacción de necesidades de los consumidores" (Bloom, Kotlher y Hayes 2004, 4).

Servicio esencial: servicios absolutamente necesarios para mantener la actividad universitaria durante un incidente disruptivo; además, deben recuperarse con brevedad en el período posterior al incidente. Estos incluyen servicios prestados por organizaciones externas y los necesarios para mantener el control de los edificios e infraestructuras con el objeto de minimizar los efectos negativos sobre la actividad universitaria.

Gestión de riesgos: es el proceso planificado, concertado, participativo e integral de reducción de las condiciones de riesgo de desastres de una comunidad, una región o un país. Implica la complementariedad de capacidades y recursos locales, regionales y nacionales y está íntimamente ligada a la búsqueda del desarrollo sostenible (PNUD 2012).

Cultura organizacional: la cultura organizacional se define como el sistema de significado compartido por los miembros, la cual distingue a una organización de las demás (Robbins y Judge 2013). 
Con base en lo anterior, se puede definir la cultura organizacional en gestión de riesgos como el sistema de significado compartido por los miembros de una empresa o institución en relación con la identificación y manejo de los riesgos que pueden afectar a la misma, el grado de importancia y compromiso de los colaboradores con este aspecto es fundamental para el desarrollo de una adecuada gestión de riesgos.

Gestión de continuidad de los servicios: proceso integral de gestión que identifica las amenazas potenciales para una organización y los posibles impactos para sus operaciones y proporciona un marco para la construcción de la resiliencia de la organización con la capacidad de una respuesta efectiva que salvaguarde los intereses clave de sus partes interesadas, así como su reputación, marca y actividades que crean valor (INTECO 2015).

Sistema de gestión de continuidad de los servicios: parte del sistema general de gestión que establece, implementa, opera, da seguimiento, revisa, mantiene y mejora la continuidad de los servicios (INTECO 2015).

Plan de continuidad: procedimientos documentados que guían a las organizaciones para responder, recuperar, reanudar y restaurar a un nivel predefinido de operación después de una interrupción. El plan de continuidad incluye los recursos, servicios y actividades necesarios para asegurar la continuidad de las funciones críticas del negocio (INTECO 2015).

\section{Referencias}

Bloom, Paul, Philip Kotlher y Thomas Hayes. 2004. Marketing de servicios profesionales. Barcelona: Paidós.

Brea, Leydi Mercedez. 2014. Factores determinates del sentido de pertenencia de los estudiantes de arquitectura de la Pontificia Universidad Católica, Madre y Maestra Santo Tomás de Aquino. Murcia: Universidad de Murcia.

Chacón, Gustavo. 2017. Gestión de cambios organizacionales. Caracas: Universidad Católica San Andrés Bello.

CR Contraloría General de la República de Costa Rica. 2020. Seguimiento de la gestión para la continuidad de los servicios públicos críticos ante la emergencia sanitaria. San José: CGR.

Espinosa Díaz, Yessica, Claudia Figueroa Rochín, Félix Lizalde Martínez y Jesuán Sepúlveda Rodríguez. 2012. Plan de continuidad académica utilizando tecnologías de información, 
comunicación y colaboración ante una contingencia en una institución de educación superior. Ciudad de México: Universidad Nacional Autónoma de México.

Fontal Hernández, Rodrigo. 2014. Modelo de gestión integral de emergencias y plan de continuidad del negocio. Palmira: Universidad Nacional de Colombia, Sede Palmira.

INTECO. 2013. INTE 22320: Seguridad de la sociedad. Gestión de emergencias. Requisitos para respuesta ante incidentes. San José: INTECO.

---. 2015. INTE/ISO 22301 Seguridad de la sociedad. Sistemas de gestión de continuidad del negocio. Requisitos. San José: INTECO.

---.2016. INTE/ISO TS 22317:2016 Seguridad de la sociedad. Sistema de gestión de continuidad del negocio. Directrices para el análisis de impacto al negocio (BIA). San José: INTECO.

---. 2018. INTE/ISO 31000:2018 Gestión del riesgo. Directrices. San José: INTECO.

INTECO y CNE. 2014. CNE-NA-INTE-DN-01 Norma de planes de preparativos y respuesta ante emergencias para centros laborales o de ocupación pública. Requisitos. San José: INTECO.

Programa de las Naciones Unidas (PNUD). 2012. Conceptos generales sobre la gestión de riesgos. Santiago: PNUD.

Robbins, Stephen P., y Timothy A. Judge. 2013. Comportamiento organizacional. Ciudad de México: Pearson.

Solano, Verónica Groskieta. s. f. "Programas de refuerzo de las habilidades sociales". Escuela Nicasio Landa. Accedido diciembre de 2020. https://bit.ly/30bsiwR.

Universidad de Costa Rica (UCR). 2018. Plan de emergencias de la UCR-SO 2018-2019. San Ramón: UCR.

---. 2019. "Sedes y recintos". Universidad de Costa Rica. Accedido septiembre de 2020. http://bit.ly/373lwht.

Unión General de Trabajadores. 2020. Prevenciones de riesgos laborales. Barcelona: Arco Libros. https://https://bit.ly/3kRrDJN. 